\title{
Evaluación Psicopedagógica e Intervención Sociocomunicativa en Niños con Trastorno de Asperger. Análisis de un Caso
}

\author{
Psychological Assessment and Socio-communicative \\ Intervention for Children with Asperger Syndrome. A Case \\ Analysis
}

\author{
Cecilia Latorre Cosculluela* y Miguel Puyuelo Sanclemente \\ Universidad de Zaragoza
}

\begin{abstract}
El proceso de evaluación de necesidades sociocomunicativas de los niños con trastorno de Asperger debe estar adecuadamente justificado con el fin de definir las líneas de intervención en contextos naturales más oportunas, aprovechando las competencias para fomentar las habilidades más deficitarias de esta población. En este trabajo se pretende llevar a cabo una evaluación psicopedagógica de un niño con síndrome de Asperger para diseñar un trabajo de intervención en el área social y comunicativa apropiado, y que tiene como finalidad última la adaptación social del niño al entorno en que se encuentra inmerso. Se adoptó para ello el método cualitativo de estudio de caso con un enfoque interpretativo que posibilitara una comprensión profunda del caso en cuestión. De los datos recogidos se derivan una serie de necesidades educativas relacionadas con la conducta adaptativa del niño en la escuela y el establecimiento de relaciones sociales. Se proponen, además, algunas orientaciones de intervención social y comunicativa para potenciar un comportamiento funcional en el contexto escolar. De igual modo que los rasgos que caracterizan a la población de niños en general difieren significativamente entre sí, el caso aquí analizado es un claro ejemplo de la heterogeneidad que se detecta entre los niños con TEA en lo que a necesidades sociales y comunicativas se refiere. La evaluación de estas dimensiones se considera de fundamental importancia, siendo competencia de los profesionales del campo de la psicología y la educación la acomodación de las pautas establecidas para estos casos, a las particularidades de cada niño.
\end{abstract}

Palabras clave: Síndrome de Asperger, Necesidades sociocomunicativas, Evaluación psicopedagógica, Intervención social y comunicativa.

\begin{abstract}
The assessment process of socio-communicative needs of children with Asperger syndrome must be properly justified in order to set the most appropriate and naturalistic lines of intervention, taking advantage of competences to promoting affected skills of this population. This paper aims to carry out a psychological assessment of a child with Asperger Syndrome in order to design an appropriate social-communicative intervention, whose ultimate goal is the social adaptation of the child to the setting in which he is included. For that it was taken the qualitative case study method with an interpretive approach that enables a deeper understanding of the case. All collected data illustrate a set of educational needs related to adaptive behavior of the child in school and establishing social relationships. Some social and communicative guidelines to promote the functional behavior of the child in the school context are also proposed. Just as defining characteristics of the population of children in general differ significantly from each other, the analyzed case here is a clear example of the diversity that id found among children with ASD in terms of social and communication needs are concerned. The assessment of these dimensions is considered a critical aspect. Educational and psychology professionals have the responsibility to adapt the guidelines that are established for such cases to the particularities of these children.
\end{abstract}

Keywords: Asperger Syndrome, Socio-communicative needs, Psychopedagogical assessment, Social and communicative intervention.

*Contacto: cecilialacos@gmail.com issn: 1989-0397

www.rinace.net/riee/

https://revistas.uam.es/riee
Recibido:

7 de noviembre de 2015

$1^{\text {a }}$ Evaluación: 20 de enero de 2016

Aceptado: $\quad 16$ de febrero de 2016 


\section{Introducción ${ }^{1}$}

Considerando la versión actualmente más consultada del Manual Diagnóstico y Estadístico de los Trastornos Mentales publicada en 2002, el DSM-IV-TR, el Trastorno de Asperger se caracteriza por una inhabilidad grave y permanente en la dimensión de interacción social y la manifestación de pautas de conducta e intereses repetitivos y limitados. El trastorno puede repercutir de manera clínicamente significativa en la competencia social y laboral, así como en otras áreas esenciales de la actividad funcional de las personas (American Psychiatric Association, 2002).

Recientemente, las continuas discrepancias entre los investigadores del tema han originado numerosas opiniones acerca de la validez de la etiqueta del síndrome. Con el fin de esclarecer esta situación, en la última versión de este manual publicada en 2013 viene establecido un continuo sintomatológico en el que se incluyen desde individuos con leves síntomas autistas hasta otros con problemas más severos, todos ellos englobados bajo la categoría de Trastorno de Espectro Autista (en adelante, TEA) (APA, 2013, citado por APA, 2014). Cabe señalar, no obstante, que este concepto ha sido recientemente cuestionado por otros autores (Tsai, 2013; Perry, 2014), quienes manifiestan su oposición a este continuo defendiendo la utilidad clínica que tienen algunos diagnósticos anteriormente utilizados en este espectro para orientar a los clínicos en la determinación del pronóstico y los planes de tratamiento.

En nuestros días, bien es sabido que uno de los desafíos más complejos que el sistema educativo debe afrontar, se relaciona con ofrecer una respuesta adecuada a las necesidades educativas de su alumnado (Lledó, Lledó y Pomares, 2011). En concreto, la atención educativa a los niños con síndrome de Asperger se considera un proceso de fundamental importancia, y tiene la finalidad de contribuir a una mejora del proceso educativo de los mismos.

Dentro del conjunto de necesidades que presenta esta población en el ámbito educativo, las alteraciones cualitativas de la interacción social se consideran un aspecto definitorio de la misma (Sansosti, 2010). Más concretamente, se manifiestan por una serie de dificultades para formar amistades verdaderas, fracaso en el uso y comprensión de pautas no verbales de comunicación, y ausencia de reciprocidad social y emocional. Por otro lado, y aunque uno de los criterios diagnósticos que se establecen en el DSM-IVTR para el síndrome de Asperger es la ausencia de un retraso del lenguaje significativo, los datos de diferentes estudios (Attwood, 2009; Etchepareborda, 2001) sugieren que el retardo precoz del lenguaje no debería ser un criterio de exclusión sino de inclusión. Finalmente, los altos niveles de rigidez mental y conductual que presentan estos niños se manifiestan a través de patrones comportamentales que conducen a una falta de interés por compartir sus conocimientos de manera recíproca (Dahle y Gargiulo, 2004). Tomando en consideración la nueva clasificación que aparece en el DSM-5, los niños que anteriormente se diagnosticaban con trastorno de Asperger, pasarían ahora a

\footnotetext{
${ }^{1}$ Este artículo ha estado financiado por fondos del Departamento de Ciencia y Tecnología del Gobierno de Aragón al grupo de investigación consolidado en Psicología: Cognición, Lenguaje, Aprendizaje y Ciclo Vital. Aspectos Evolutivos y Educativos.
} 
considerarse dentro de ese abanico de TEA, más concretamente de alto funcionamiento.

Los criterios que se acaban de describir suponen, en la mayoría de los casos, un obstáculo que puede interferir en la interacción social con sus iguales. El hándicap más significante de este espectro se localiza en el funcionamiento social (Adams, Green, Gilchrist y Cox, 2002). Incluso en aquellos casos de niños con un grado de funcionalidad no significativamente afectado, las dificultades en el área social siguen constituyéndose como una de las necesidades educativas más características (Paul, Miles, Chuba y Volkmar, 2009).

La población de niños con TEA de alto funcionamiento necesita que, desde los contextos educativos, se les enseñe cómo funcionan las rutinas y los diferentes modos de establecer interacciones con otros compañeros, de tal manera que logren manejar su propia conducta y comprender las reglas sociales que rigen las conversaciones (Barquero, 2007). A causa de su poca habilidad para deducir, de forma intuitiva, los pensamientos, planes e intenciones de otras personas, los niños con síndrome de Asperger y, en general, con TEA, requieren además que se les aporten los recursos necesarios para comprender conductas, anticipar reacciones y relacionarse apropiadamente con los demás. En muchas ocasiones, y de acuerdo con Barquero (2007), el problema no es la poca interacción social, sino más bien la eficacia de ésta.

Puesto que son niños que asimilan y retienen mejor la información que les llega por la vía visual, las estrategias que adopten esta fuente de entrada de datos serán las más adecuadas para comprender mejor a este alumnado y lograr así la consecución de objetivos educativos (Lledó et al., 2011). En el área social, y utilizando el método de enseñanza que se acaba de mencionar, será necesario que estos niños se encuentren con tareas que persigan, a grandes rasgos, la modificación de patrones de conductas no adecuados sustituyéndolos por comportamientos sociales coherentes con cada situación.

El perfil comunicativo de estos niños de alto funcionamiento varía desde algunos que presentan marcadas alteraciones cualitativas y cuantitativas en su habilidad para comprender y transmitir la información, hasta otros que cuentan con un desarrollo del lenguaje verbal apropiado para su edad (Shriver, Allen y Mathews, 1999). En estos últimos casos siguen detectándose ciertas peculiaridades en su proceso comunicativo que interfieren en su desempeño social y lingüístico y que resultan condicionantes directos de su funcionamiento sociocomunicativo tanto en el ámbito escolar como en otros contextos de interacción.

En lo que respecta a los aspectos formales del lenguaje, autores como González Carbajal (2002) señalan una conservación de los mismos en los niños de alto funcionamiento, pudiendo presentar no obstante un "patrón específico de desarrollo de habilidades morfosintácticas y semánticas fuertemente asociado a este trastorno" (Martín Borreguero, 2005, p.117). En términos de comportamiento pragmático, estos niños ven alterada su comprensión del lenguaje figurativo, el sarcasmo y el humor, la prosodia, la expresión facial y otras formas de lenguaje no verbal que intervienen en la función comunicativa (Williams, Goldstein, Kojkowski y Minshew, 2008). Consecuentemente, aparece en estos niños la necesidad de aprender habilidades de relación social que, desde una perspectiva pragmática del lenguaje, se concibe como la capacidad de actuación social (González Carbajal, 2002). 
Tal y como se observa en el estudio de Paul et al. (2009), los aspectos conversacionales más significativamente afectados y que deberían ser tomados en consideración en todo planteamiento de intervención, son la cantidad de información proporcionada en la conversación para satisfacer las necesidades del oyente, la capacidad para mantener y para responder a las señales sociales. Otras áreas incluyen la capacidad para iniciar y mantener un discurso espontáneo e inferir los estados mentales de los demás. Nuevamente, los apoyos visuales serán los que permitan al niño aprender, a través de una enseñanza explícita, a expresar sus ideas y explicarse correctamente, a respetar los turnos de palabra y a comprender las expresiones faciales ligadas a las emisiones verbales de otras personas.

En el área comunicativa, otra de las necesidades educativas de la población de niños con síndrome de Asperger (o con TEA de alto funcionamiento, si atendemos a la nueva clasificación del DSM-5) se relaciona con su capacidad narrativa. Las intervenciones que se planteen presentarán un trabajo sobre este aspecto, ayudando al niño a no emitir información irrelevante, a seguir una secuencia temporal y a elaborar narraciones en las que se incluyan referencias a los estados mentales de los personajes (González Carbajal, 2002). Diversas investigaciones, entre ellas la de Losh y Capps (2003) han encontrado relaciones entre el rendimiento en tareas de falsa creencia de niños con TEA y la frecuencia de descripciones de estados mentales y afectivos de los personajes de las historias narrativas. Precisamente por ello, el trabajo de intervención con estos niños incluirá, necesariamente, tareas en las que se vea implicada la capacidad de atribución de estados mentales.

Analizando las consecuencias educativas que el síndrome tiene para el niño que lo padece, destaca su falta de interés para participar en los juegos sociales, tendiendo por ende a un juego repetitivo y aislado (Frontera, 2010; Lledó et al. 2011). Cuando se incorporan a la escuela comienzan a aparecer conflictos de adaptación al aula con alteraciones conductuales, una tendencia a evitar interacciones sociales espontáneas y dificultades para mantener conversaciones sencillas. Si bien manifiestan la necesidad de ser aceptados socialmente por sus compañeros, en ausencia de tales interacciones estos niños presentan sentimientos de aislamiento y depresión (Bonete, Vives, Fernández, Calero y García, 2010). Por tanto, desconocen cómo interactuar adecuadamente debido a su ajustada comprensión social y emocional.

Desde el ámbito psicoeducativo, la necesidad de seguir planteando el análisis de las necesidades que aparecen en niños con síndrome de Asperger (o TEA de alto funcionamiento) es absolutamente evidente, con la finalidad de dar cabida a una atención adaptada a las características de cada niño y de su entorno. Así, un trabajo explícito sobre el ámbito social y comunicativo puede suscitar mejoras considerables siempre y cuando, en la línea de lo expuesto por Monfort (2009), los contenidos se extraigan de las necesidades reales del niño y se introduzcan después en su contexto.

La pretensión de este estudio es presentar a los educadores, psicopedagogos y demás profesionales que trabajan a diario con alumnos con TEA, una ejemplificación del proceso de evaluación psicopedagógica y de la propuesta de intervención más oportuna dadas las necesidades educativas de un caso particular. En un primer momento se consideró necesario analizar el perfil de habilidades sociales y lingüísticas del niño, de tal manera que los datos recogidos sirvieran como punto de partida para el diseño de una intervención desde el contexto de escolaridad ordinaria. La integración de toda la información permitió una descripción del funcionamiento actual del niño y de sus 
necesidades así como la determinación de las estrategias necesarias para estimular su desarrollo y aprendizaje.

\section{Método}

El presente trabajo de investigación se elaboró a través de una metodología cualitativa utilizando el estudio de caso, presentando éste un carácter descriptivo-interpretativo, pues pretende describir los diferentes factores que ejercen influencia en el fenómeno objeto de estudio (Martínez Carazo, 2006). La intención final del mismo es generalizar desde un punto de vista analítico, es decir, utilizar este trabajo para ilustrar y representar una teoría ya existente.

La elección del caso se justifica por dos criterios fundamentales, a saber: en primer lugar, por el aliciente de conocer, específicamente, las necesidades sociales y comunicativas de un niño que todavía no ha alcanzado la etapa de Educación Primaria; $\mathrm{y}$, en segundo lugar, por la posibilidad de acceso tanto al niño en cuestión como al entorno escolar en que se encuentra inmerso.

\subsection{Unidad de análisis}

La unidad de análisis es situacional $(\mathrm{n}=1)$, es decir, un único sujeto con unas características peculiares alrededor del cual se organiza la recogida de datos y las orientaciones de intervención. El caso de estudio es un niño escolarizado, desde hace tres años, en un colegio público de enseñanza ordinaria, y que actualmente se encuentra en el nivel de $3^{\circ}$ de Educación Infantil. Hasta la fecha, no ha sufrido enfermedades destacables ni se encuentran bajo tratamiento médico. Además, su evolución respecto a los aprendizajes escolares es propia de su edad.

Para la elaboración de este estudio, tanto familia como escuela debían presentar las autorizaciones correspondientes. Previamente, la tutora del aula del niño informó de que, aparentemente, el niño presentaba una capacidad cognitiva adecuada a su edad. La maestra advierte también una serie de comportamientos extraños con el resto de compañeros de la clase que se traducen en ciertas dificultades para acercarse a interaccionar con ellos. Además de presentar ecolalias y un contacto ocular episódico, tiene un interés obsesivo por las letras y los números.

\subsection{Instrumentos}

Con el propósito de asegurar un mayor rigor y objetividad en los resultados del estudio, fueron varios los instrumentos de recogida de datos seleccionados (Stake, 1998). Para analizar el área social, se utilizó una escala de evaluación de la conducta adaptativa. Por otro lado, la detección de necesidades derivadas de la dimensión lingüística y comunicativa se realizó por medio de un instrumento estandarizado, una lista de chequeo y un registro de observación durante la resolución de tareas que ponían en juego las capacidades narrativas del niño.

Para identificar las dimensiones lingüísticas que podrían estar afectadas se utilizó el $P L O N-R$ (Prueba de Lenguaje Oral de Navarra) (Aguinaga, Armentia, Fraile, Olangua y Uriz, 2004), un instrumento elaborado para detectar problemas lingüísticos a edades tempranas. La tutora del aula del niño cumplimentó una de las grandes escalas de evaluación de la conducta adaptativa en el actual panorama internacional, el $A B A S-I I$ (Oakland y Harrison, 2013). Permite una evaluación directa de los principales déficits que presentan los niños con TEA, incluidas las habilidades sociales y de comunicación. 
Se analizaron además los resultados obtenidos tras la administración del CCC-2 (Children's Communication Checklist) (Bishop, 2003) a la tutora del niño.

En cuanto a la evaluación de habilidades narrativas, se utilizaron tres series de secuencias con apoyo visual en base a las que el niño debía crear un relato a través del lenguaje oral. Este análisis se justifica, de acuerdo con Baixauli, Roselló y Miranda (2004), por las dificultades observadas en niños con TEA para utilizar términos mentalistas, pudiendo además crear historias narrativas con una cantidad pobre de información y una desestructuración de las ideas del discurso.

\section{Resultados}

La información recabada se analizó por medio de la técnica de la triangulación de fuentes, un principio que aporta racionalidad, de tal manera que las categorías examinadas son: el síndrome de Asperger, las necesidades sociales y comunicativas, y el caso propiamente dicho. La fiabilidad de la investigación se aseguró contando con una base de datos que sustenta el estudio de casos, de tal modo que, con los datos recogidos, un nuevo investigador pudiera repetir el estudio y llegar a conclusiones similares.

Una de las necesidades educativas más evidentes que se detectan tras la cumplimentación del ABAS-II se localiza en la dimensión de interacción social del niño, siendo de hecho el ámbito más significativamente afectado que aparece en esta escala. Causada probablemente por estas dificultades primarias en el establecimiento de interacciones sociales, se aprecia además la necesidad de plantear una intervención en lo que respecta a la competencia comunicativa del niño y su funcionamiento en el ámbito escolar. Así, y en términos generales, el resultado que se desprende en cuanto a la conducta adaptativa general (CAG) sitúa al niño en un nivel "bajo".

La información obtenida en el cuestionario CCC-2 ofrece datos relevantes sobre su desarrollo lingüístico. Unos resultados que orientan acerca del planteamiento alrededor del cual debe girar la intervención psicoeducativa en el ámbito escolar, y que parte de las necesidades detectadas en las escalas de uso del contexto, relaciones sociales, intereses e iniciación inadecuada. Si bien en sintaxis y semántica no se aprecian déficits relevantes, el nivel de funcionamiento está por debajo de lo que se esperaría para un niño de su edad.

Las puntuaciones extraídas en las distintas dimensiones lingüísticas que incluye el PLON-R sirven también de indicadores acerca de los posibles ámbitos hacia los que urge plantear el trabajo de intervención. En las dimensiones de "forma" y "contenido" del lenguaje, el niño se sitúa en un rango de "necesita mejorar", mientras que en la dimensión de "uso" correspondiente al ámbito pragmático, se encuentra ubicado en un nivel de "retraso". El ámbito pragmático se establece, por tanto, como el área prioritaria de intervención dentro de la competencia comunicativa del niño.

En cuanto a la producción narrativa utilizando las secuencias de imágenes, las observaciones y anotaciones realizadas señalan ciertos aspectos de la coherencia narrativa que se ven alterados durante la producción verbal y que se traducen en un conjunto de necesidades que orientarán el trabajo de intervención. Dado que no establece relaciones de significado entre los elementos que componen las secuencias narrativas, uno de los requisitos bajo los que se plantearán las posteriores tareas, se relaciona con la utilización de apoyos visuales que dirijan y orienten la elaboración de las historias. De acuerdo con los resultados obtenidos en esta prueba de carácter 
cualitativo, este niño precisará además un trabajo explícito sobre atribución de estados mentales y emociones a los demás, pues sus dificultades de comprensión se incrementan cuando la historia adquiere un carácter más emocional.

Una vez examinada la información se presentan, en el siguiente espacio, las líneas de intervención en habilidades psicosociales y lingüísticas que, dadas las necesidades del niño, deberían guiar el trabajo en el centro educativo en que se encuentra escolarizado.

\subsection{Intervención en habilidades psicosociales y lingüísticas}

Para atender a las dificultades de niños con unas características similares a las del caso al que se refiere este trabajo (síndrome de Asperger o, de acuerdo con la nueva versión del DSM, TEA de alto funcionamiento), es necesario disponer de apoyo de pedagogía terapéutica orientado a ofrecerles la ayuda necesaria para comprender las diferentes rutinas, aceptar los cambios e interactuar con los compañeros. Durante la etapa de escolarización se requiere además apoyo logopédico con el fin de potenciar el desarrollo de los distintos ámbitos del lenguaje y, especialmente, de la comunicación (Frontera, 2010).

Tras conocer las necesidades educativas de este niño, lo cual supone un verdadero reto para muchos profesionales, se planteó la respuesta educativa encaminada a desarrollar al máximo sus habilidades sociales y comunicativas con vistas a la aplicación de los saberes adquiridos. Dada la gran diversidad existente dentro del espectro autista respecto al grado de severidad y el modo en que los síntomas se manifiestan, resultaría inapropiado aplicar orientaciones de intervención de carácter general sin llevar a cabo una individualización significativa (Frontera, 2012). Se diseñaron así una serie de orientaciones que se basarían en los siguientes principios de intervención que Lledó et al. (2011) proponen:

$\checkmark$ Enseñar de forma explícita a través de instrucciones y apoyos visuales.

$\checkmark$ Estructurar el entorno de aprendizaje para que se convierta en predecible.

$\checkmark$ Anticipar los posibles cambios y proponer diferentes alternativas.

En lo que respecta al ámbito social, los objetivos que se establecen para este caso son, por un lado, apropiarse de las normas sociales que rigen las conversaciones y, por otro, comprender situaciones sociales específicas que encuentra confusas a través de una enseñanza explícita de habilidades de inicio, mantenimiento y finalización de conversaciones ajustadas a cada situación. Se establecen, por ende, las siguientes orientaciones específicas para la intervención en el área psicosocial:

$\checkmark$ Una herramienta de gran utilidad para enseñar al niño a enfrentarse a situaciones sociales son los guiones (scripts) sociales. Consisten, de acuerdo con Frontera (2010, p.220), en "una descripción explícita de la secuencia de pasos a seguir en una situación específica, indicando el tipo de comportamiento que se espera de ellos".

$\checkmark$ Es conveniente mostrarle, de la manera más objetiva y perceptible posible, las maneras de llevar a cabo una interacción social exitosa trabajando cuestiones como "por qué sucede algo", "cuáles son las consecuencias que se producen en el interlocutor cuando no se respeta su turno de palabra” y demás aspectos relacionados con situaciones de interacción. En este sentido, las "conversaciones en forma de historieta" de Carol Gray (1998) pueden resultar una herramienta de gran utilidad a partir de las que podemos personalizar las situaciones que queramos enseñarle. Teniendo en cuenta los resultados 
obtenidos en el ABAS-II, este material puede resultar de gran utilidad para trabajar en el niño aquellos aspectos que se encuentran más deteriorados como, por ejemplo, las actividades del día a día en el aula y la escuela.

$\checkmark$ Role-playing: este tipo de metodología de trabajo con el niño supondrá, de acuerdo con autores como De la Iglesia y Olivar (2005) la toma de conciencia de "qué" y "cómo" decir y hacer en un buen número de situaciones sociales. Estos aprendizajes ofrecerán al niño una base sobre la que ir construyendo un repertorio de comportamientos acordes a cada circunstancia específica.

$\checkmark$ Para trabajar la inferencia de estados mentales y lograr una mejor comprensión de las situaciones sociales, pueden utilizarse dibujos en los que aparezcan imágenes de distintas situaciones en las que los personajes se ven inmersos. Un material de gran utilidad es el elaborado por Monfort y Monfort (2002), donde aparecen diversas tareas destinadas a estimular los procesos mentalistas de niños con dificultades. Algunos ejemplos son los que aparecen las Figuras 1 y 2.

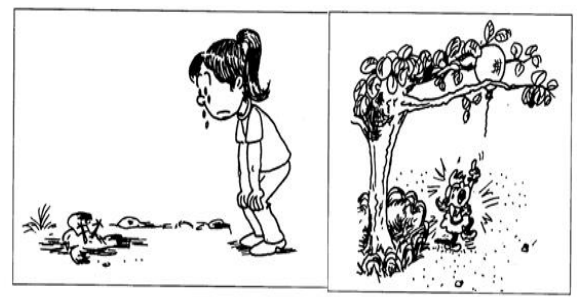

Figura 1. Ilustraciones extraídas del material "En la Mente"

Fuente: Monfort y Monfort (2002).

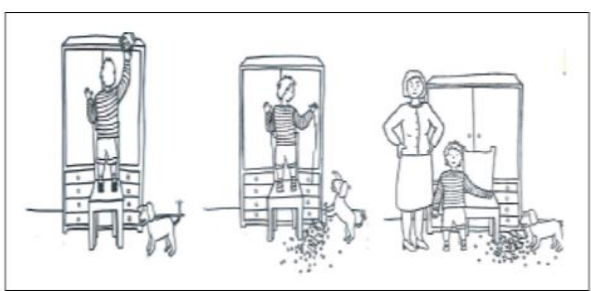

Figura 2. Viñeta para trabajar emociones secundarias

Fuente: Rodríguez, García, Gorriz y Regal (2002).

$\checkmark$ Una vez se hayan trabajado los aspectos anteriormente comentados, conviene generalizar estos aprendizajes en contextos de interacción con otros niños, en forma de pequeño o gran grupo. El entrenamiento sistemático en habilidades sociales ayuda a desarrollar habilidades comunicativas y resolver problemas en situaciones de interacción social (Griffin, Griffin, Fitch, Albera y Gingras, 2006). Pero no olvidemos que "el aprendizaje será más efectivo si se le proporcionan al niño experiencias significativas que se produzcan dentro de contextos de interacciones sociales" (González Carbajal, 2002, p.97).

$\checkmark$ Rutinas diarias: El orden secuencial de los eventos y el establecimiento de rutinas estables fomentarán en el niño la sensación de orden y la habilidad para predecir los acontecimientos cotidianos (Frontera, 2012), ayudando así al niño a evitar situaciones de estrés y ansiedad.

Por otro lado, el objetivo inicial de cualquier intervención en el desarrollo comunicativo va dirigido a "promover el aprendizaje de formas adecuadas de regulación de otras personas, alternativas al empleo de formas de comunicación inadecuadas" (Gortázar, 2003, p.183). En este caso, la meta de la intervención sobre los aspectos lingüísticos y comunicativos es lograr que el niño pueda comunicarse mejor con sus interlocutores habituales. Por ello, la intervención logopédica en niños con TEA de alto funcionamiento se centra fundamentalmente en la enseñanza del uso social adecuado del lenguaje. Las orientaciones específicas más adecuadas para este caso son las que a continuación aparecen expuestas: 
$\checkmark \quad$ Los juegos de rol y las dramatizaciones constituyen una ventajosa herramienta para trabajar diversos aspectos de la pragmática, el discurso y la conversación, ámbitos en los que este niño manifiesta considerables dificultades. Esta metodología permitirá además trabajar los movimientos corporales, los gestos, la prosodia y los sentimientos propios y ajenos.

$\checkmark$ Para trabajar específicamente las habilidades lingüísticas pragmáticas, se puede utilizar el apoyo visual de secuencias que conforman una historia y que el niño deberá construir y narrar utilizando el lenguaje oral. En este caso, la intervención en este aspecto es esencial, pues las dificultades en la narración pueden limitar el acceso a la interacción con otros afectando significativamente a la competencia sociocomunicativa del niño. Nuevamente, el material "En la Mente" de Monfort y Monfort (2002) puede emplearse también para trabajar el área pragmática del lenguaje del niño, más concretamente la compresión de preguntas y de situaciones de mentira y broma.

$\checkmark$ Las tareas de lectura pueden resultar un instrumento muy interesante para favorecer el discurso narrativo de este niño. De acuerdo con González Carbajal (2002), y a partir de la lectura de una pequeña historia utilizando los apoyos necesarios, se puede dialogar sobre el contenido, seleccionando los aspectos relevantes del mismo y extrayendo las ideas principales.

$\checkmark \quad$ Las conversaciones en forma de historieta de las que hablábamos en el apartado anterior han demostrado también tener una gran eficacia para trabajar habilidades comunicativas. Usando dibujos, "bocadillos" de pensamiento, símbolos y diferentes colores, se pueden mostrar y enseñar al niño diferentes aspectos tales como el turno de palabra, los cambios en el tema de conversación, las clarificaciones y la evitación de comentarios irrelevantes.

$\checkmark \quad$ Las actividades de comunicación referencial resultan una herramienta muy útil para trabajar situaciones comunicativas (González Carbajal, 2002). El niño tiene que contar o describir algo a otra persona sin ver el objeto en cuestión a describir.

$\checkmark \quad$ Una vez que dejemos atrás la enseñanza explícita de estas situaciones y normas sociales, pasaremos a trabajarlas en un contexto más funcional de interacción: practicándolo con el adulto en una situación simulada. Durante esta actividad el especialista reforzará las actitudes adecuadas del niño y ofrecerá modelos para sustituir aquellos intentos erróneos de establecimiento de interacción. Como soporte a este proceso pueden utilizarse apoyos visuales de fotografías, dibujos y tarjetas, que fomentarán la atención y comprensión del niño durante los intercambios comunicativos (Frontera, 2012).

$\checkmark$ El nivel más complejo para esta actividad es el que se refiere a la puesta en práctica de las habilidades aprendidas en un contexto real de interacción con sus iguales. Se aprovecharán situaciones en las que un grupo de niños estén jugando y se animará al niño a que se acerque a ellos y ponga en práctica lo que se ha trabajado.

Todas las actuaciones que se han descrito en este trabajo se apoyan en las necesidades psicoeducativas que tiene este niño con síndrome de Asperger, y tienen como objetivo prioritario una intervención que conduzca al desarrollo y optimización de habilidades de interacción social para la mejora de las relaciones interpersonales. Además, la función de los educadores, psicopedagogos, orientadores y logopedas es crucial en este proceso, debiendo contar todos ellos con una formación competente para colaborar en 
el proceso de evaluación, en la búsqueda de alternativas de respuesta y en la valoración del proceso educativo (Frontera, 2010).

\section{Discusión}

Pese a ser un niño que sigue un patrón normalizado de evolución, presenta las necesidades educativas específicas del diagnóstico de espectro autista. Consecuentemente, y de igual modo que se encuentra en las investigaciones de estudio de caso de Jurado y Bernal (2011) y Vives y Ascanio (2012), las áreas más afectadas son las relacionadas con la interacción social, el lenguaje y la comunicación. En esta línea, los resultados que Saulnier y Klin (2007) obtienen en su estudio con niños con síndrome de Asperger y Autismo de Alto Funcionamiento señalan, igual que lo encontrado en este trabajo, la necesidad de realizar evaluaciones de las habilidades comunicativas y sociales de cada niño para determinar del grado de funcionalidad adaptativa en contextos de la vida real.

En el caso escogido para la elaboración de este estudio, y coincidiendo con los resultados del estudio de Jurado y Bernal (2011), las necesidades más notables derivadas de la evaluación psicopedagógica se localizan en el ámbito pragmático del lenguaje, que se encuentra estrechamente relacionado con el área social. Por el contrario, las dificultades de comprensión social y lingüística descubiertas en este niño discrepan con los hallazgos del trabajo de Lin y Chiang (2014), quienes sugieren que no existe una diferencia significativa entre niños con trastorno de Asperger y niños con desarrollo típico en cuanto a comprensión se refiere.

Por otro lado, las puntuaciones obtenidas en el ABAS-II confirman estas necesidades sociocomunicativas. De acuerdo con los resultados del estudio de validez del ABAS-II (Oakland y Harrison, 2013, p.159) con una muestra de niños autistas, los resultados aquí encontrados están en consonancia con los de estos autores, aunque en el caso del que se ocupa este estudio se obtiene un grado de funcionalidad de conducta adaptativa general (CAG) más elevado que en el grupo de estos autores.

Las revisiones llevadas a cabo por autores como Johnson et al. (2007) también señalan un conjunto de características que están presentan en niños diagnosticados dentro del espectro autista y que deben ser interpretadas, desde el contexto escolar, como necesidades a tener en consideración por los profesionales que van a trabajar con estos niños. En concordancia con lo encontrado en este caso, estos autores determinan la aparición de dificultades conversacionales y de interacción comunicativa que deben ser valoradas en la intervención posterior. En cuanto a las necesidades derivadas del uso del contexto, en otros trabajos como el de Loukusa et al. (2007) también se concluye la pertinencia de ofrecer a estos niños apoyo en la interpretación de emisiones comunicativas en función de la situación en que se encuentran inmersos.

De manera complementaria, el análisis de las habilidades narrativas ha permitido descubrir que la inferencia de aspectos que no se manifiestan de manera explícita en la historia, es deficitaria en este niño lo que, según Whyte y Nelson (2015), es un aspecto relativamente frecuente en niños con TEA. También en la revisión de estudios sobre inferencia pragmática en individuos con Asperger y AAF que Loukusa y Moilanen (2009) llevan a cabo, se demuestra para ambas condiciones la presencia de dificultades y, en consecuencia, la necesidad de iniciar una intervención, en aquellas tareas donde se ponen en práctica habilidades de inferencia pragmática y entendimiento de 
intencionalidad, ofreciendo como posible explicación al respecto los déficits detectados en Teoría de la Mente que tradicionalmente se han atribuido a esta población. Por estas razones, algunos de los contenidos planteados en el proceso de intervención tienen por finalidad potenciar, en la medida de lo posible, todos estos aspectos.

Los resultados que se acaban de exponer deben interpretarse con cautela pues, siguiendo lo expuesto por Van der Paelt, Warreyn y Roeyers (2014), la edad cronológica es un significante predictor del desarrollo del lenguaje que, además, se encuentra estrechamente relacionado con la competencia sociocomunicativa de cada niño. Por ello, cabe la posibilidad de que, con el transcurso de los años de escolaridad, estos niños con TEA de alto funcionamiento evolucionen considerablemente en todas estas dimensiones en las que ahora se localizan necesidades específicas de apoyo psicoeducativo.

Si bien una investigación como la propuesta puede limitar la generalización de resultados, se constata la importancia de seguir planteando el análisis de las realidades que suponen la presencia de diversas necesidades y características en el ámbito escolar. De acuerdo con los resultados del estudio de Vives y Ascanio (2012), las intervenciones que parten del análisis de las necesidades sociocomunicativas de los niños tienen la virtud de lograr generalizar, en muchas ocasiones, otras conductas que no son tratadas directamente. Por ende, tan importante como planificar una o varias sesiones de evaluación de las competencias del niño, es el planteamiento de una intervención ajustada a sus necesidades. La adaptación de estas intervenciones puede tener, indudablemente, efectos claramente favorecedores para su desarrollo comunicativo y social, tal y como se muestra en el trabajo de Adams (2003) con niños con Trastorno Pragmático del Lenguaje.

Este enfoque, aunque comience con un trabajo individual, deberá ir orientado a la aplicación en contextos naturales y con interlocutores cotidianos, potenciando los entornos comunicativos y el papel activo del niño (González Carbajal, 2002). Desde el punto de vista de cualquier profesional responsable de implementar una intervención, lo fundamental es adaptar las orientaciones generales a las particularidades de cada niño y su entorno. Se enseña de manera explícita lo que de forma natural no se ha podido aprender, y se adapta esa enseñanza a cada individuo en particular (Monsalve, 2002), muestra de lo cual es el caso aquí estudiado. El alcance de este tipo de intervenciones puede comprobarse también en estudios como el de Millar, Light y Schlosser (2006) con personas con trastornos del desarrollo, encontrándose una mejora significativamente positiva en la producción comunicativa y lingüística.

El apoyo de los educadores, terapistas y familia, así como el diseño de intervenciones adecuadas, puede ayudar a los niños con Trastorno de Asperger (y, en general, a los niños con TEA) a afrontar más eficazmente sus dificultades y funcionar de un modo más independiente en el contexto familiar, escolar y comunitario (Griffin et al. 2006). Por ende, es tarea de los profesionales especializados en el campo de la psicología y la educación acomodar las pautas de trabajo a las individualidades de cada niño. Este asesoramiento pedagógico incluido dentro de un proyecto inclusivo de intervención será el que, de acuerdo con Darretxe y Sepúlveda (2011), facilite a estos niños el acceso a los aprendizajes en las mejores condiciones. 


\section{Referencias}

Adams, C., Green, J., Gilchrist, A. y Cox, A. (2002). Conversational behaviour of children with Asperger syndrome and conduct disorder. Journal of Child Psychology and Psychiatry, 43(5), 679-690. doi:10.1111/1469-7610.00056.

Adams, C. (2003). Intervention for developmental pragmatic language impairments. Aula Abierta, 82, 79-96.

Aguinaga, G., Armentia, M., Fraile, A., Olangua, P. y Uriz, N. (2004). Prueba de Lenguaje Oral de Navarra-Revisada (PLON-R). Pamplona: Fondo de Publicaciones del Gobierno de Navarra.

American Psychiatric Association. (2002). Trastornos generalizados del desarrollo. En DSM$I V-T R$, Manual diagnóstico y estadísticos de los trastornos mentales (pp. 79-97). Barcelona: Masson.

American Psychiatric Association. (2014). Trastornos generalizados del desarrollo. En DSM-5, Manual diagnóstico y estadísticos de los trastornos mentales (pp. 125-148). Buenos Aires: Panamericana.

Attwood, T. (2009). ¿Qué es el síndrome de Asperger? El diagnóstico. En Guía del síndrome de Asperger (pp. 19-89). Barcelona: Paidós.

Barquero, M. (2007). Síndrome de Asperger. Guía para padres de familia y educadores. San José: Vicerrectoría de Acción Social, Universidad de Costa Rica.

Bishop, D. V. M. (2003). Children's Communication Checklist (CCC-2). Recuperado de https://moodle.usal.es/

Baixauli, I., Roselló, B. y Miranda, A. (2004). Evaluación de las dificultades pragmáticas. Estudio de casos. Revista de Neurología, 38(1), 69-79.

Bonete, S., Vives, M. C., Fernández, A., Calero, M. D. y García, M. B. (2010). Potencial de aprendizaje y habilidades sociales en escolares con el trastorno de Asperger. Behavioral Psychology/Psicología Conductual, 18(3), 473-490.

Dahle, K. B. y Gargiulo, R. M. (2004). Understanding Asperger disorder: A primer for early childhood educators. Early Childhood Education Journal, 32(3), 199-203. doi:10.1023/b:ecej.0000048973.89175.a3.

Darretxe, L. y Sepúlveda, L. (2011). Estrategias educativas para orientar las necesidades educativas de los estudiantes con síndrome de Asperger en aulas ordinarias. Electronic Journal of Research in Educational Psychology, 9(24), 869-892.

De la Iglesia, M. y Olivar, J. S. (2005). Orientaciones para la intervención psicopedagógica en niños con síndrome de Asperger y autismo de alto nivel de funcionamiento cosgnoscitivo. Puertas a la lectura, 18, 70-83.

Etchepareborda, M. C. (2001). Perfiles neurocognitivos del espectro autista. Revista de Neurología Clínica, 2(1), 175-192.

Frontera, M. (2010). Cuarta propuesta: El desafío del desarrollo. Alumnos con síndrome de Aspeger. Necesidades y respuesta educativa. En E. Arrimadas, S. Ramos y A. DíazGüemes (Coords.), Desafíos de la diferencia en la escuela (pp. 179-252). Madrid: Edelvives.

Frontera, M. (2012). Intervención: Principios y programas psicoeducativos. En M. A. Martínez y J. L. Cuesta (Coords.), Todo sobre el autismo (pp. 22 1-270). Tarragona: Altaria.

González Carbajal, A. (2002). Intervención comunicativa en autismo de alto funcionamiento y síndrome de Asperger. En J. Martos y M. Pérez Juliá (Coords.), Autismo: Un enfoque orientado a la formación en logopedia (pp. 85-106). Valencia: Nau Llibres. 
Gortázar, P. (2003). Intervención comunicativa en casos con autismo: Estado de la cuestión. Presentación de un caso clínico. En F. Alcantud (Coord.), Intervención psicoeducativa en niños con Trastornos Generalizados del Desarrollo. Madrid: Pirámide.

Griffin, H. C., Griffin, L. W., Fitch, C. W., Albera, V. y Gingras, H. (2006). Educational interventions for individuals with Asperger syndrome. Intervention in School and Clinic, 41(3), 150-155. doi:10.1177/10534512060410030401

Johnson, C. P. y Myers, S. M. (2007). Identification and evaluation of children with Autism Spectrum Disorders. Pediatrics, 120(5), 1183-1215. doi:10.1542/peds.2007-2361.

Jurado, P. y Bernal, D. (2011). El alumno afectado con síndrome de Asperger en el aula ordinaria. Revista de Educación Inclusiva, 4(2), 29-46.

Lin, Y. H. y Chiang, H. M. (2014). Language comprehension of children with Asperger's disorder and children with autistic disorder. Research in Autism Spectrum Disorders, 8(7), 767-774. doi:10.1016/j.rasd.2014.03.018.

Lledó, A., Lledó, G. y Pomares, J. (2011). Las necesidades del alumnado con síndrome de Asperger: orientaciones y propuestas desde una innovación inclusiva. En R. Roig Vila y C. Lavene (Eds.), La práctica educativa en la sociedad de la información. Innovación a través de la investigación (pp. 243-254). Alcoy: Marfil.

Losh, M. y Capps, L. (2003). Narrative ability in high-functioning children with autism or Asperger's syndrome. Journal of Autism Developmental Disorders, 33(3), 239-251.

Loukusa, S., Leinonen, E., Kuusikko, S., Jussila, K., Mattila, M. L., Ryder, N., Ebeling, H. y Moilanen, I. (2007). Use of context in pragmatic language comprehension by children with Asperger syndrome or high-functioning autism. Journal of Autism Developmental Disorders, 37(6), 1049-1059. doi:10.1007/s10803-006-0247-2.

Loukusa, S. y Moilanen, I. (2009). Pragmatic inference abilities in individuals with Asperger syndrome or high-functioning autism. A review. Research in Autism Spectrum Disorders, 3(4), 890-904. doi:10.1016/j.rasd.2009.05.002.

Martín Borreguero, P. (2005). Perfil lingüístico del individuo con síndrome de Asperger: Implicaciones para la investigación y la práctica clínica. Revista de Neurología, 41(1), $115-$ 122.

Martínez Carazo, P. C. (2006). El método de estudio de caso. Estrategia metodológica de la investigación científica. Pensamiento y Gestión, 20, 165-193.

Millar, D. C., Light, J. C. y Schlosser, R. W. (2006). The impact of augmentative and alternative communication intervention on the speech production of individuals with developmental disabilities: A research review. Journal of Speech, Language, and Hearing Research, 49(2), 248-264. doi:10.1044/1092-4388(2006/021).

Monfort, M. y Monfort, I. (2002). En la mente: Un soporte gráfico para el entrenamiento de las habilidades pragmáticas en niños. Madrid: Entha Ediciones.

Monfort, M. (2009). Comunicación y lenguaje: Bidireccionalidad en la intervención en niños con trastorno de espectro autista. Revista de Neurología, 48(2), 53-56.

Monsalve, C. (2002). Comunicación y lenguaje en autismo: Claves para el buen trabajo de un logopeda. En J. Martos y M. Pérez Juliá (Coords.), Autismo: Un enfoque orientado a la formación en logopedia (pp. 65-83). Valencia: Nau Llibres.

Oakland, T. y Harrison, P. (2013). ABAS-II. Sistema para la evaluación de la conducta adaptativa. Madrid: TEA Ediciones. 
Paul, R., Miles, S., Chuba, H. y Volkmar, F. (2009). Conversational behaviors in youth with high-functioning ASD and Asperger syndrome. Journal of Autism Developmental Disorders, 39(1), 115-125. doi:10.1007/s10803-008-0607-1.

Perry, R. (2014). Asperger's disorder on life support. Journal of Autism Developmental Disorders, 44(8), 2072-2073. doi:10.1007/s 10803-014-2067-0.

Rodríguez, N., García, E. M., Gorriz, A. B. y Regal, R. (2002). ¿Cómo se estudia el desarrollo de la mente? Recuperado de http://www.uji.es/bin/publ/edicions/jfi8/psi/20.pdf

Sansosti, F. J. (2010). Teaching social skills to children with autism spectrum disorders using tiers of support: A guide for school-based professionals. Psychology in the Schools, 47(3), 257-281. doi:10.1002/pits.20469.

Saulnier C. A. y Klin, A. (2007). Brief report: Social and communication abilities and disabilities in higher functioning individuals with autism and Asperger syndrome. Journal of Autism Developmental Disorders, 37(4), 788-793. doi:10.1007/s10803-006-0288-6.

Stake, R. E. (1998). La triangulación. En Investigación con estudio de casos (pp. 94-104). Madrid: Morata.

Shriver, M. D., Allen, K. D. y Mathews, J. R. (1999). Effective assessment of the shared and unique characteristics of children with autism. School Psychology Review, 28(4), 538-558.

Tsai, L. Y. (2013). Asperger's disorder will be back. Journal of Autism Developmental Disorders, 43(12), 2914-2942. doi:10.1007/s 10803-013-1839-2.

Van der Paelt, S., Warreyn, P. y Roeyers, H. (2014). Social-communicative abilities and language in preschoolers with autism spectrum disorders: Associations differ depending on language age. Research in Autism Spectrum Disorders, 8(5), 518-528. doi:10.1016/j.rasd.2014.01.010.

Vives, M. C. y Ascanio, L. (2012). Resultados del tratamiento en un caso con trastorno de Asperger. Behavioral Psychology, 20(2), 401-419.

Whyte, E. M. y Nelson, K. E. (2015). Trajectories of pragmatic and nonliteral language development in children with autism spectrum disorders. Journal of Communication Disorders, 54, 2-14. doi:10.1016/j.jcomdis.2015.01.001.

Williams, D. L., Goldstein, G., Kojkowski, N. y Minshew, N. (2008). Do individuals with high functioning autism have the IQ profile associated with nonverbal learning disability? Research in Autism Spectrum Disorders, 2(2), 353-361. doi:10.1016/j.rasd.2007.08.005. 